\title{
The current status of checkpoint inhibitors in metastatic bladder cancer
}

\begin{abstract}
For many decades, no significant improvements could be achieved to prolong the survival in metastatic bladder cancer. Recently, systemic immunotherapy with checkpoint inhibitors (anti-PD-L1/anti-CTLA-4) has been introduced as a novel treatment modality for patients with metastatic bladder cancer. We conducted a systematic review according to the PRISMA statement for data published on the clinical efficacy of checkpoint inhibitors in metastatic bladder cancer. Clinical efficacy of anti PD-L1 therapy was investigated in prospective trials in a total of 155 patients. Patients with positive expression for PD-L1 tended towards better overall response rates (ORR) compared to those with negative expression (34/76 vs 10/73, 45 vs $14 \% ; \mathrm{p}=0.21)$. Among patients with PD-L1 positive tumors, those with non-visceral metastases exhibited significantly higher ORR compared to those with visceral metastases (82 vs $28 \% ; \mathrm{p}=0.001$ ). For anti-CTLA4 therapy, there were no data retrievable on clinical efficacy. Although data on clinical efficacy of checkpoint inhibitors in metastatic bladder cancer are currently limited, the efficacy of these drugs might depend mainly on the metastatic volume and immune system integrity. Patients with PD-L1 positive tumors and non-visceral metastases seem to derive the highest benefit from therapy.
\end{abstract}

Keyword: Bladder cancer immunotherapy; Checkpoint inhibitor; Ipilimumab; Nivolumab; Pembrolizumab 